\title{
Les produits de l'anesthésie 5e édition
}

\author{
Xavier Sauvageon, Pierre Viard, Jean-Pierre Tourtier. Arnette, John Libbey Eurotext, \\ Paris 2015. ISBN 978-2-7184-1384-6
}

\author{
Marco Julien, MD
}

Received: 30 November 2015 / Accepted: 7 December 2015/Published online: 15 December 2015

(c) Canadian Anesthesiologists' Society 2015

À l'ère des téléphones intelligents et des applications mobiles, le petit livre de référence que l'on traine précieusement dans la poche du sarrau s'apparente de plus en plus à une espèce en voie d'extinction. Ceci étant dit, dans le domaine de la pharmacologie, rares sont les applications en langue française et tout aussi rares sont celles qui s'adressent spécifiquement au domaine de l'anesthésiologie. C'est ainsi l'objectif que se fixe, depuis sa première édition en 1997, le livre «Les produits de l'anesthésie » : être, pour citer sa préface, « [un] ouvrage qui fait partie de nos « instruments » de travail et que nous laissons au bloc opératoire ou en salle de réveil parce qu'il répond à nos préoccupations quotidiennes ».

Ce petit livre de poche de moins de deux cents pages se présente sous la forme de fiches concises de quelques pages rassemblant les éléments clés à connaitre sur chacun des médicaments inclus. Ceux-ci sont regroupés en dix chapitres: anesthésiques intraveineux, anesthésiques volatils, analgésiques morphiniques, curares, anesthésiques locaux, solutés de remplissage vasculaire, antidotes, atropine, dantrolène et médicaments sympathomimétiques. Un chapitre sur l'utilisation de l'oxygène et un dernier sur la relation entre les médicaments anesthésiques et les porphyries et l'hyperthermie maligne complètent l'ouvrage. Chaque fiche est organisée en style télégraphique et aborde la présentation physique du médicament, ses indications, la posologie en fonction de l'usage prévu, ses contre-indications, les interactions médicamenteuses et métaboliques, sa pharmacocinétique et sa

M. Julien, MD ( $\square)$

Université de Montréal, Montréal, QC, Canada

e-mail: marco.julien@umontreal.ca pharmacodynamique, ses effets indésirables et les conséquences et traitements du surdosage. Ce format de mise en page simple, clair et «droit au but » remplit avec succès l'objectif d'offrir un outil de consultation rapide et efficace.

La qualité de l'information retrouvée dans les différentes fiches est globalement satisfaisante. Il aurait cependant été intéressant d'en savoir plus sur les sources ayant servi à créer le livre, ce dernier ne comportant aucune référence formelle. Pour le lecteur nord-américain, un certain nombre de choix éditoriaux mettent en évidence l'origine européenne de l'ouvrage et quelques différences entre les pratiques de chaque côté de l'Atlantique. Par exemple, les noms commerciaux et les formulations sont parfois distincts de ce que l'on retrouve au Canada. Si la présentation du thiopental demeure probablement de mise malgré son absence prolongée du marché nord-américain, le gamma-hydroxybutyrate de sodium n'y est pas utilisé du tout. Quant à l'usage du dropéridol en neuroleptanesthésie, sa description est maintenant limitée à un rappel historique dans les livres de référence. À l'inverse, l'absence d'une fiche sur la dexmédétomidine, dont l'utilisation est de plus en plus répandue et les indications de plus en plus nombreuses, se fait remarquer. On note la description de l'utilisation de quelques médicaments, comme le propofol et le rémifentanil, en anesthésie intraveineuse à objectif de concentration («target-controlled infusion»), une technique malheureusement peu disponible au Canada mais qui aurait avantage à se développer.

Tel que mentionné précédemment, le style télégraphique emprunté par l'ouvrage sert bien l'objectif d'en faire une référence rapide et facile d'accès mais laisse peu de place à l'approfondissement de certaines affirmations qui, sans contextualisation, peuvent laisser perplexe. Par exemple, la 
contre-indication du propofol en anesthésie chez l'enfant de moins de trois ans et chez la femme enceinte nous semble pouvoir difficilement être affirmée sans plus de détail, tout comme la contre-indication absolue du rémifentanil en anesthésie obstétricale et celle de la kétamine en présence d'une coronaropathie. Également, si la grande majorité de l'information étudiée nous apparait exacte et appropriée, quelques données soulèvent des questions. Par exemple, la dose d'entretien de l'anesthésie générale au propofol de 6 à $12 \mathrm{mg} \cdot \mathrm{kg}^{-1} \cdot \mathrm{h}^{-1} i v$ (équivalent à 100 à $200 \mu \mathrm{g} \cdot \mathrm{kg}^{-1} \cdot \mathrm{min}^{-1} i \mathrm{v}$ ) parait un peu faible, et est de façon étonnante la même dose qui est rapportée quelques lignes plus loin pour la sédation de complément (pour laquelle elle semble plutôt élevée).

Ainsi, sur la quatrième de couverture, on peut lire que ce guide "s'adresse prioritairement aux jeunes internes en anesthésie réanimation et aux élèves des écoles d'infirmiers anesthésistes ». Il est fort à parier que cette clientèle cible - et son équivalent dans le système canadien - trouvera dans cet ouvrage une référence francophone utile, bien construite et facile à consulter qui répondra à une partie de ses besoins. D'autant plus que le lecteur visé appartient à la jeune génération, il nous semble que le développement d'une version électronique pour appareil mobile aurait été un avantage significatif. En ce qui concerne le clinicien plus aguerri ou le résident avancé dans sa formation, il est peu probable que «Les produits de l'anesthésie, $5^{\mathrm{e}}$ édition» puisse répondre aux besoins plus approfondis de connaissance pharmacologique, mais il pourra servir de rappel occasionnel que les plus nostalgiques pourront même garder dans une poche de leur sarrau.

Conflit d'intérêt Aucun. 